\title{
Acute Liver Failure Secondary to Metastatic Breast Cancer in a Patient with Ulcerative Colitis
}

\author{
Luisa Leite Barros ${ }^{1}$, Bianca Pocopetz Facas ${ }^{1}$, Camila Cunha Gonzaga Lima ${ }^{1}$, Paula Emília Rivas Farez ${ }^{2}$, \\ Alberto Queiroz Farias ${ }^{1}$
}

Departments of ${ }^{1}$ Gastroenterology and Hepatology and ${ }^{2}$ Pathology, Hospital das Clínicas, School of Medicine, University of São Paulo, São Paulo, SP, Brazil.

\section{Corresponding Author: \\ Dr Luisa Leite Barros}

Email: luisa.barros@hc.fm.usp.br

This is an Open Access article distributed under the terms of the Creative Commons Attribution License (creativecommons.org/ licenses/by/3.0).

Received : November 14, 2020

Accepted : March 1, 2021

Published : April 20,2021

\begin{abstract}
Background: Acute liver failure (ALF) induced by metastatic disease is rare and has never been described in conjunction with the inflammatory bowel diseases (IBD) setting. Case Report: Our case report is of a 57-year-old patient diagnosed with ulcerative colitis (UC) admitted to the emergency department with jaundice, abdominal pain and encephalopathy for one week. She had been treated with thiopurine and anti-tumor necrosis factor alpha (anti-TNF) for the past four years prior to the admission and denied previous known liver disease. A computed tomography (CT) scan demonstrated an enlarged liver with heterogeneous hepatic parenchymal attenuation and diffuse fat deposition, suggesting acute hepatitis. Common causes of ALF were excluded and the immunosuppressive therapy was immediately withdrawn. While in intensive care, the patient experienced acute deterioration of hepatic encephalopathy and died of multiorgan failure. The liver autopsy was consistent with ALF secondary to metastatic undifferentiated breast carcinoma. Conclusion: This is the rare case report of an UC patient that developed ALF secondary to breast cancer..
\end{abstract}

Keywords: Abdominal Pain, Breast Neoplasms, Hepatic Encephalopathy, Jaundice, Ulcerative Colitis.

\section{Introduction}

Inflammatory bowel diseases (IBD), Crohn's disease (CD) and ulcerative colitis (UC), are chronic immune-mediated disorders characterized by a chronic course of relapse and remission. IBD affects young productive adults and usually requires lifelong treatment. For the past decade, mucosal healing has emerged as an important therapeutic goal, although it has additionally increased the use of immunosuppressive drugs, such as thiopurines and biologics [1].

Recent IBD guidelines have highlighted the risk of adverse events and of new onset extra-intestinal malignancy related to immunosuppression, particularly in the elderly population [2]. Furthermore, the persistent inflammatory state in IBD is a well-recognized risk factor for cancer [3]. Previous studies have calculated a pooled prevalence of breast cancer of $6 \%$ in IBD subjects [4]. Diffuse metastatic breast carcinoma is rare and associated with high mortality rates. On the other hand, liver involvement is frequently reported amongst IBD patients occurring as superimposed primary sclerosing cholangitis (PSC), autoimmune hepatitis (AIH) or non-alcoholic fatty liver disease (NAFLD) $[5,6]$. Drug-induced liver injury (DILI) can occur in patients treated with 5-aminosalicylates or immunosuppressants. Nonetheless, acute liver failure (ALF) is uncommon and related to previous anti-TNF exposure [7,8]. ALF is a clinical condition marked by jaundice, coagulopathy 
and hepatic encephalopathy. It occurs within 28 weeks of initial symptomatic onset, typically in patients without pre-existing chronic liver disease [9]. The most common causes of ALF are direct drug toxicity, acute viral hepatitis, autoimmune hepatitis, ischemic injury and metabolic diseases. Liver neoplastic infiltration is rare and occurs in up to $1.5 \%$ of cases, with a diffuse pattern of metastatic carcinoma $[10,11]$.

Herein we describe an UC patient that developed ALF secondary to metastatic breast carcinoma, while being treated with combined azathioprine and adalimumab.

\section{Case Report}

A 57-year-old female UC patient presented at the emergency department with recent symptoms of jaundice, dark urine, abdominal pain, nausea, vomiting and encephalopathy. She also reported fatigue, reduced appetite and progressively worsening lower limb edema for a week prior to hospitalization. She had no fever or chills and had no sick contacts. The patient had been well and was in deep remission for the past year. Past medical history was consistent with severe pancolitis treated with mesalamine, azathioprine and adalimumab for 5 years. She had no previous liver disease and denied episodes of hepatitis, alcohol abuse or drug allergy. The patient's family history was not significant for any co-morbidities.

On admission, the patient was dehydrated, hypotensive and had deeply icteric skin and sclerae. There were no prior signs of chronic liver disease, such as spider angiomas and palmar erythema. She presented a distended abdomen with marked hepatomegaly and hypoactive bowel sounds without evidence of ascites. Cardiac and respiratory examinations were otherwise normal. Neurologic examination showed mild lethargy and asterixis. Physical examination of her left breast showed a mass of $5.0 \mathrm{~cm}$ by palpation, without inflammatory skin changes or nipple discharge, suspicious for malignancy.
Laboratory testing revealed normal hemoglobin, leukocytosis and a platelet count of $55 \mathrm{ml} / \mathrm{mm}^{3}$. C-reactive protein was $31 \mathrm{mg} / \mathrm{dL}$ and serum creatinine $1.9 \mathrm{mg} / \mathrm{dL}$. She had elevated liver enzymes with an AST of $595 \mathrm{U} / \mathrm{L}$ and ALT of $105 \mathrm{U} / \mathrm{L}$. Liver function tests showed a total bilirubin of $6 \mathrm{mg} / \mathrm{dL}$, direct bilirubin $3.8 \mathrm{mg} /$ $\mathrm{dL}$ and albumin of $1.8 \mathrm{~g} / \mathrm{dL}$. Prothrombin time was prolonged with an international normalized ratio (INR) of 7.0. Serum fibrinogen was $132 \mathrm{mg} /$ $\mathrm{dL}$ and the factor $\mathrm{V}$ activity was $5 \%$. Her venous ammonia concentration was $141 \mu \mathrm{mol} / \mathrm{L}$. An extensive workup diagnosis excluded hepatotropic virus infections, such as hepatitis A, B, C, EpsteinBarr and cytomegalovirus. Autoantibodies ANA, SMA and AMA were negative. Serum IgG was $7.3 \mathrm{~g} / \mathrm{L}$, while IgA, IgM, $\alpha 1$-antitrypsin and ceruloplasmin were normal. Autoimmune and metabolic panel tests were all in the normal range and failed to show a precise etiology of liver impairment. An abdominal CT scan revealed an enlarged liver, heterogeneous hepatic parenchymal attenuation suggesting acute hepatitis with diffuse fat deposition. There was no bile-duct dilatation, gallbladder distention or cholelithiasis. The main portal and hepatic veins were unobstructed. The thoracoabdominal transition demonstrated a left breast mass, suspicious for malignancy [Fig.1].

The patient was admitted to the intensive care unit for hemodynamic stabilization and required mechanical ventilation to prevent cerebral

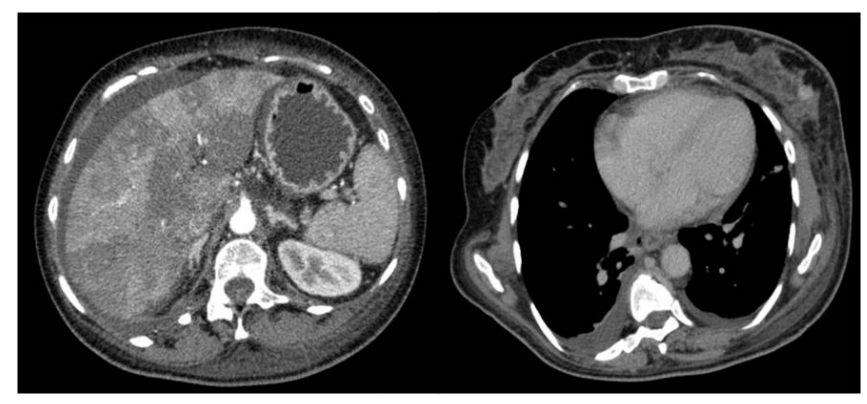

Fig.1: Contrast-enhanced axial CT scan of the abdomen showed a heterogeneous hepatic parenchymal attenuation with diffuse fat deposition and a hyper-enhanced spiculated mass with irregular margins in the left breast. 
edema. She was treated with broad-spectrum antibiotics, fresh frozen plasma and platelet transfusion. A presumptive diagnosis of DILI was made and 5-aminosalicylate, immunosuppressant and the anti-TNF were immediately withdrawn. She was not a liver transplant candidate due to the suspected concomitant breast cancer. Despite supportive care, the patient experienced clinical deterioration, grade IV hepatic encephalopathy, bleeding diathesis and rapid progression to ALF. Within two days she developed cardiac arrest and died of multiorgan failure. On post-mortem examination the final diagnosis was ALF with extensive liver damage secondary to a metastatic undifferentiated carcinoma and neoplastic vascular emboli. Immunohistochemistry analysis confirmed a primary breast carcinoma [Fig.2,3] with negative expression of estrogens, progesterone and HER2 receptors.

\section{Discussion}

This report has described a fatal presentation of ALF secondary to metastatic breast cancer in an immunosuppressed UC patient. In the case presented, the patient had no known prior cirrhosis or previous alcohol abuse. Serological tests for infectious hepatitis and autoimmune disease were negative. A preemptive diagnosis of DILI was made considering the temporal relation with drug exposure, lack of other causes of liver injury or laboratory changes. The final diagnosis was made on autopsy.

Hepatic involvement may be present in $25 \%$ of IBD patients. PSC and AIH are the most common causes of associated liver disease and generally present in a chronic course. DILI caused by TNF- $\alpha$ antagonists is a recognized complication of IBD treatment. Previously, Ghabril et al. have described 34 cases of DILI in both IBD or rheumatological subjects and infliximab was the most common agent implicated. Of these, 67\% tested positive for anti-nuclear or smooth muscle antibodies and presented autoimmune features

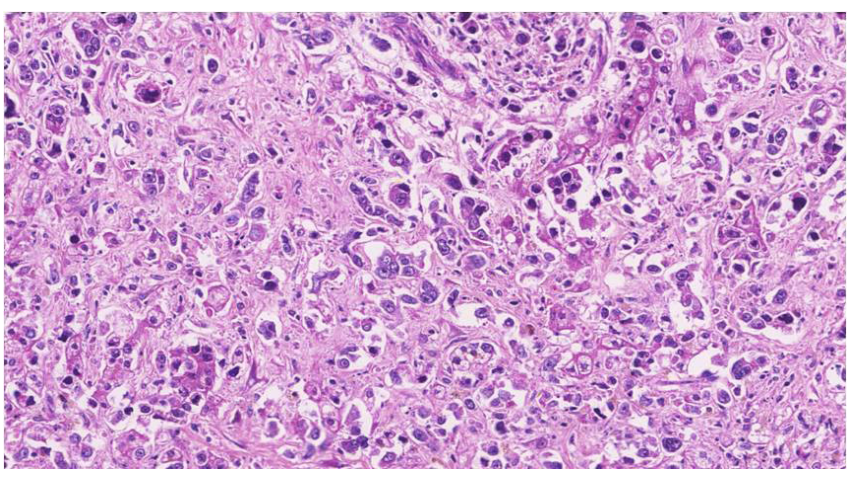

Fig.2: Photomicrograph of poorly cohesive cells, showing atypical mitoses, nuclear pleomorphism and increased cytoplasm-nucleus ratio (H\&E, 100x).

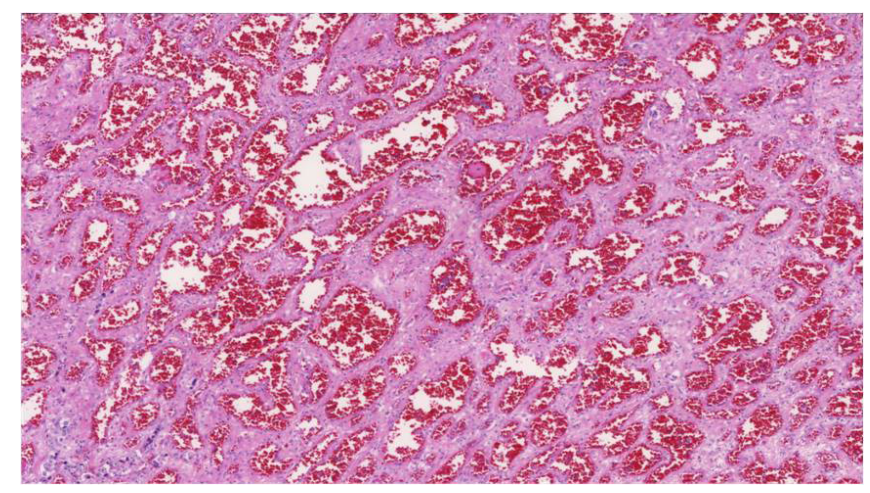

Fig.3: Photomicrograph showing the dilated and ectasic vessels of the neoplasia (H\&E, 20x).

on the liver biopsy. One patient with pre-existent cirrhosis required liver transplantation and no deaths were observed [7]. In this scenario, the prognosis is favorable after drug discontinuation. Nevertheless, ALF has been reported in 9 cases treated with anti-TNF. In the study by Kok et al, 5 of them had rheumatologic diseases and 4 patients had UC. Infliximab was the most common antiTNF- $\alpha$ drug and only one patient was treated with adalimumab. They observed an extended latency period between initial drug exposure and ALF indicating a possible idiosyncratic drug reaction. Six of the patients required a liver transplant. When performed the liver biopsies demonstrated a cholestatic-hepatitis predominance, without a clear autoimmune pattern [8]. It is difficult to determine the specific drug toxicity in these patients and the diagnosis is often based upon exclusion. 
IBD patients, especially UC, are at higher risk for intestinal and extra-intestinal malignancies when compared to the general population. Preliminary data have shown a strong association between thiopurine and the risk of specific cancers, such as non-melanoma skin cancer and lymphoma [12]. In the French IBD research, the adjusted hazard ratio for lymphoproliferative disorders is $5 \%$ in patients exposed to thiopurines compared to naive subjects. Few studies have reported that CD patients with family history of breast cancer are at higher risk for malignancy. These authors have suggested a common genetic and/or environmental role in the pathogenesis of both $\mathrm{CD}$ and breast carcinoma. Further studies are required to confirm this association. In rare cases, hepatic failure can occur as a result of metastatic disease. Rowbotham et al. have analyzed over 4,020 cases of ALF for 18 years and have calculated a prevalence of $0.44 \%$ of malignant infiltration [13]. Most commonly attributed to hematologic malignancies especially in non-Hodgkin lymphomas, but also associated with solid tumors such as lung and breast cancer.

In the literature, there are several reports of undiagnosed metastatic liver disease presenting with ALF in patients with breast cancer. Agarwal et al. described a similar case presented as acute variceal hemorrhage and portal hypertension secondary to intra-sinusoidal infiltration of breast adenocarcinoma. This patient had no prior cancer diagnosis [14]. Furthermore, Ogata et al. have reported two cases of ALF in this circumstance. Both patients had a diagnosis of breast cancer and the CT scan had not detected liver metastasis. These authors suggested that a liver biopsy should be performed after 2 weeks of elevated liver enzymes, when frequent etiologies have been excluded [15]. Few authors have suggested a pathophysiological mechanism of hepatic impairment in metastatic cancer. Goswani et al. have shown that liver cell necrosis would be secondary to an invasion of hepatic vessels by tumor cells [16]. Nazario et al. highlighted the role of cellular adhesion molecular expression, such as E-cadherin and CD44. ALF would occur in patients that have lost expression of these proteins, with subsequent tumor detachment and cell infiltration [17].

In IBD population, this is the first description of ALF secondary to breast cancer. Diffuse liver metastasis is rare and difficult to diagnose. It is not detectable during routine radiographic tests. These patients have a limited survival rate since liver transplantation is contraindicated and chemotherapy offers a prohibitive toxicity when hepatic failure is established.

\section{Conclusion}

Our report documents a rare case of ALF induced by metastatic breast cancer in an immunosuppressed UC patient. While there is no specific recommendation for breast cancer surveillance in IBD patients it is important to reinforce that active screening could potentially avoid a negative outcome related to immunosuppression.

Contributors: LLB: manuscript writing, patient management; BPL, CCGL: manuscript editing, patient management; PERF, AQF: critical inputs into the manuscript. LLB will act as guarantor. All authors approved the final version of this manuscript and are responsible for all aspects of this study. Funding: None; Competing interests: None stated.

\section{References}

1. Peyrin-Biroulet L, Sandborn W, Sands BE, Reinisch W, Bemelman W, Bryant RV, et al. Selecting therapeutic targets in inflammatory bowel disease (STRIDE): Determining therapeutic goals for treat-to-target. Am J Gastroenterol. 2015;110(9):1324-1338.

2. Annese V, Beaugerie L, Egan L, Biancone L, Bolling C, Brandts C, et al. European evidence-based consensus: Inflammatory bowel disease and malignancies. J Crohns Colitis. 2015;9(11):945-965.

3. Axelrad JE, Lichtiger S, Yajnik V. Inflammatory bowel disease and cancer: The role of inflammation, immunosuppression, and cancer treatment. World J Gastroenterol. 2016;22(20):4794-4801.

4. Biancone L, Armuzzi A, Scribano ML, Castiglione F, D'Inca R, Orlando A, et al. Cancer Risk in inflammatory bowel disease: A 6-year prospective multicenter nested case-control IG-IBD study. Inflamm Bowel Dis. 2020;26(3):450-459. 
5. Chao CY, Battat R, Al Khoury A, Restellini S, Sebastiani $\mathrm{G}$, Bessissow T. Co-existence of non-alcoholic fatty liver disease and inflammatory bowel disease: A review article. World J Gastroenterol. 2016;22(34):7727-7734.

6. Koller T, Galambosova M, Filakovska S, Kubincova $\mathrm{M}$, Hlavaty T, Toth J, et al. Drug-induced liver injury in inflammatory bowel disease: 1-year prospective observational study. World J Gastroenterol. 2017;23(22):4102-4111.

7. Ghabril M, Bonkovsky HL, Kum C, Davern T, Hayashi $\mathrm{PH}$, Kleiner DE, et al. Liver injury from tumor necrosis factor-alpha antagonists: analysis of thirty-four cases. Clin Gastroenterol Hepatol. 2013;11(5):558-64 e3.

8. Kok B, Lester ELW, Lee WM, Hanje AJ, Stravitz RT, Girgis S, et al. United States Acute Liver Failure Study G. Acute liver failure from tumor necrosis factor-alpha antagonists: Report of four cases and literature review. Dig Dis Sci. 2018;63(6):1654-1666.

9. Stravitz RT, Lee WM. Acute liver failure. Lancet. 2019;394(10201):869-881.

10. Lee WM. Etiologies of acute liver failure. Semin Liver Dis. 2008;28(2):142-152.

11. Rich NE, Sanders C, Hughes RS, Fontana RJ, Stravitz $\mathrm{RT}$, Fix $\mathrm{O}$, et al. Malignant infiltration of the liver presenting as acute liver failure. Clin Gastroenterol Hepatol. 2015;13(5):1025-1028.
12. Lemaitre M, Kirchgesner J, Rudnichi A, Carrat F, Zureik M, Carbonnel F, et al. Association between use of thiopurines or tumor necrosis factor antagonists alone or in combination and risk of lymphoma in patients with inflammatory bowel disease. JAMA. 2017;318(17):1679-1686.

13. Rowbotham D, Wendon J, Williams R. Acute liver failure secondary to hepatic infiltration: a single centre experience of 18 cases. Gut. 1998;42(4):576-580.

14. Agarwal K, Jones DE, Burt AD, Hudson M, James OF. Metastatic breast carcinoma presenting as acute liver failure and portal hypertension. Am J Gastroenterol. 2002;97(3):750-751.

15. Ogata T, Kikawa Y, Ogata M, Satake H, Hatachi Y, Yasui H. Acute liver failure with diffuse liver metastasis from breast cancer, not detected by computed tomography: 2 case reports. Case Rep Oncol. 2018;11(3):699-704.

16. Goswami R, Babich M, Farah KF. Occult breast malignancy masquerading as acute hepatic failure. Gastroenterol Hepatol (NY). 2011;7(1):62-65.

17. Nazario HE, Lepe R, Trotter JF. Metastatic breast cancer presenting as acute liver failure. Gastroenterol Hepatol (NY). 2011;7(1):65-66. 